\title{
Política de Reconstrucción y Desplazamiento: el caso de las familias de bajos ingresos del centro de Talca ${ }^{1}$
}

\author{
Reconstruction Policy and Displacement: The case of low income families of \\ inner Talca city
}

Francisco Letelier Troncoso ${ }^{2}$

Alejandra Rasse Figueroa ${ }^{3}$

Recibido: 18 de agosto de 2016

Aceptado: 16 de diciembre de 2016

\section{Resumen}

El 27 de febrero de 2010 (27F) un terremoto 8.8 afectó la zona centro sur de Chile. La Región del Maule fue una de las más afectadas y su capital, Talca, sufrió la destrucción de parte importante de su centro, que constituye $20 \%$ de su superficie urbana construida. En este vivían muchas familias no propietarias, en condiciones de vulnerabilidad. A partir de la revisión de datos secundarios, documentos oficiales e información de prensa, este trabajo explora la manera en que actores gubernamentales, privados y de la sociedad civil actuaron frente al problema de las familias no propietarias del centro de Talca. Dentro del análisis, se sostiene que el no ser propietarios, su condición socioeconómica y la prevalencia de la jefatura de hogar femenina y de adultos mayores, constituyeron factores de riesgo que los hicieron vulnerables a perder su localización. Este riesgo no fue abordado por las políticas públicas, que carecieron de perspectiva territorial y consideración del derecho a la localización. Se concluye que más allá de las debilidades de los instrumentos, el mayor obstáculo estuvo en la ausencia de una política pública que permitiera abordar los efectos del terremoto adecuadamente e incluir las necesidades y expectativas de la sociedad civil.

Palabras clave: desplazamiento, política de vivienda, reconstrucción.

\begin{abstract}
The 8.8 magnitude earthquake (27F) affected the south central zone of Chile on 27 of February 2010. The Maule Region was one of the most affected and its capital, Talca, suffered the destruction of an important part of its center, which constitutes $20 \%$ of its built urban area. Many non-proprietary families lived there with vulnerability conditions. Based on the review of secondary data, official documents and press information, this paper explores how government, private and civil society sectors acted in the face of the problem of non-owner families in the center of Talca. From the analysis, it is argued the lack of ownership, socioeconomic status and the prevalence of female household heads and older adults, were risk factors that made them vulnerable to losing their location. This risk condition was not addressed by public policies, with a lack of territorial perspective and consideration of the right to location. It is concluded that apart from a weak master planning system, the biggest obstacle was the absence of a public policy that would not allow to deal with the effects of the earthquake properly and to include both the needs and expectations of civil society.
\end{abstract}

Keywords: displacement, housing policy, reconstruction.

\footnotetext{
${ }^{1}$ Este estudio fue desarrollado en el marco del trabajo de los autores en el Centro de Estudios Urbanos y Territoriales para la Región del Maule CEUT, y el Centro de Desarrollo Urbano Sustentable CEDEUS FONDAP 15110020.

${ }^{2}$ Universidad Católica del Maule. Contacto: fletelier@ucm.cl

${ }^{3}$ Pontificia Universidad Católica de Chile, CEDEUS. Contacto: arasse@uc.cl
} 


\section{Introducción}

El casco histórico de la ciudad de Talca es un territorio en que confluyen usos y habitantes diversos. Sus barrios, generados hace décadas por autoconstrucción al alero de programas de vivienda económica y obrera, con el tiempo han ido generando diversidad social (producto de procesos de movilidad social y urbana), dando forma a un sector actualmente heterogéneo. El estándar urbano de la época, con grandes plazas y espaciosas calles, así como la proximidad al centro comercial y de servicios de la ciudad, definen el área como un sector que ofrece una buena calidad de vida para sus habitantes. Alrededor de la mitad de estos son familias de bajos ingresos, que ven en su localización un importante activo y una forma eficaz de integración social (Rasse y Letelier, 2013). Esto se hace evidente cuando se compara su situación con la de aquellas familias populares que residen en la periferia de la ciudad, en un entorno socialmente homogéneo y alejados de las oportunidades urbanas (Letelier y Boyco, 2013).

Con el terremoto del 27 de febrero de 2010, más de la mitad de las viviendas del centro quedaron seriamente dañadas o totalmente destruidas (Rasse y Letelier, 2013), dejando una gran cantidad de familias damnificadas. Estas familias no eran homogéneas entre sí: algunas correspondían a propietarios de las viviendas; otras eran allegadas, y también había familias arrendatarias. Asimismo, algunas disponían de recursos propios, mientras otras (encabezadas por mujeres o por adultos mayores) estaban en situación de mayor vulnerabilidad (Instituto Nacional de Estadísticas, INE, 2002). Tenían en común el arraigo al barrio, tanto por los vínculos de vecindad, como por la relevancia funcional de su localización en sus actividades cotidianas (Valdivieso, 2013).

La respuesta de la política pública consistió en brindar a los damnificados la posibilidad de optar a los mismos instrumentos de acceso a la vivienda utilizados en tiempos ordinarios, que consistían básicamente en subsidios para viviendas nuevas en sitios nuevos, o en el mismo sitio (en el caso de quienes tenían la propiedad del suelo). También existieron subsidios para la reparación de viviendas (Valdivieso, 2013).

Como puede observarse, existe una tensión entre el arraigo y la importancia del territorio para los damnificados y la propuesta desterritorializada de política de reconstrucción. Mientras los damnificados buscaban no solo una vivienda, sino una vivienda en su barrio, la política estaba orientada a proveer de manera eficaz un techo a quienes lo habían perdido. Los inmobiliarios, por su parte, buscaron proveer viviendas allí donde también pudieran generar negocio inmobiliario (por valorización del suelo), por lo que prefirieron la construcción de viviendas en sitios nuevos. En este marco, el objetivo de este trabajo es conocer la forma en que se desenvolvieron y tensionaron los intereses de los distintos actores involucrados en el proceso de reconstrucción en la ciudad de 
Talca. Se hipotetiza que la falta de perspectiva territorial en la política de vivienda, junto con la vulnerabilidad de los grupos "sin tierra" y el protagonismo del sector inmobiliario, se conjugaron teniendo como resultado el desplazamiento de las familias de menores recursos del centro a la periferia de la ciudad de Talca.

\section{Terremoto, reconstrucción y desplazamiento}

Para efectos de este trabajo, resulta importante relevar la relación entre desastres naturales (en este caso un terremoto) y el desplazamiento de residentes originales, así como el vínculo de esto con las políticas de reconstrucción.

El terremoto genera una aceleración de los procesos de obsolescencia de las áreas centrales. En la práctica, la destrucción de las edificaciones aumenta la brecha de renta, al liberar al suelo de su uso original (Inzulza y Díaz, 2016). Esto hace más rápido el proceso de gentrificación de áreas centrales.

En este marco, lo que se plantea como reconstrucción de un barrio, puede ser entendido en realidad como un proceso de gentrificación vía construcción de nuevos conjuntos, que incluye tanto la llegada de habitantes de mayor nivel socioeconómico, como el desplazamiento de quienes residían en las edificaciones originales. Resulta interesante incorporar acá la noción de desplazamiento también en su dimensión de declive del sentido de lugar (en tanto dis-place-ment), ya que las nuevas tipologías de vivienda incorporadas se unen a la destrucción generada por el terremoto en un cambio radical del paisaje del barrio (Davidson y Lees, 2010).

Esta situación, sin embargo, no debe ser entendida como un resultado mecánico (Inzulza y Díaz, 2016). Por el contrario, intervienen en esto la forma en que se haya planteado la política de reconstrucción y el rol que adopten al respecto los actores involucrados. En el caso del terremoto de México, por ejemplo, los afectados por el sismo se volvieron actores sociales relevantes, muy organizados, que lograron buscar apoyos y forzar al gobierno a actuar en una cierta dirección (Ducci, 1986). A partir de un análisis comparativo, Inzulza y Díaz (2016) concluyen que en un escenario neoliberal los actores privados cobran un protagonismo mucho más acentuado en la reconstrucción que en otros contextos.

Esta relevancia de los actores privados en la reconstrucción de la Región del Maule post 27F ha sido relevada por diversos estudios (Concha y Rasse, 2014; Inzulza, 2014; Inzulza y Díaz, 2016; Rasse y Letelier, 2013). De acuerdo a estos autores, este liderazgo del mercado ha llevado a una falta de pertinencia territorial de las soluciones generadas, y a la pérdida de identidad de los 
barrios. Asimismo, han detectado indicios de gentrificación en el surgimiento de conjuntos habitacionales orientados a hogares de mayores ingresos que los residentes tradicionales.

Por otra parte, también es posible imaginar escenarios alternativos a partir de la discusión respecto de los elementos que se vuelven relevantes en un urbanismo que hace frente a los desastres naturales. Así, Wamsler (2007), señala la importancia de la coordinación entre los diversos actores involucrados, de enfocarse en el desarrollo más que en la emergencia, y de establecer el liderazgo desde el nivel local, para poder integrar vivienda, planificación urbana y gestión del riesgo. Davoudi (2012) cuestiona la pertinencia de fijar como objetivo el retorno a lo normal, cuando en realidad la catástrofe revela desigualdades y procesos sociales en curso que pueden no ser deseables para los habitantes de una ciudad, lo que obliga a pensar en instrumentos de planificación innovadores, creativos, y propositivos desde lo local. En esta misma línea, Shaw (2012) plantea que la planificación, al enfrentarse a los desastres, debería estar preparada para una transformación innovadora y no quedarse en la práctica habitual, ya que esto solo permite resolver los problemas del pasado (Davoudi, 2012). Todo lo anterior implica pensar los procesos de reconstrucción desde su potencial de transformación y las capacidades locales, evitando modelos que acentúan la dependencia, limitan las alternativas de las personas, profundizan la desigualdad de los más débiles, fuerzan a la gente a salir, y en suma, no crean beneficios para la comunidad local (Lechner et al., 2016).

En suma, existe una clara relación entre desastres naturales y desplazamiento, pero esta debe ser entendida como contingente, en la medida en que la configuración de los actores locales y las orientaciones de política pueden modificar este vínculo de forma importante. En este marco de discusión, presentamos el caso del proceso de reconstrucción de la ciudad de Talca tras el terremoto del $27 \mathrm{~F}$.

\section{Metodología}

Para lograr el objetivo propuesto, se ha trabajado con un diseño de estudio de caso, específicamente, la reconstrucción de Talca posterremoto del $27 \mathrm{~F}$. El caso ha sido construido combinando diversas fuentes de información: estadísticas oficiales (datos censales, informes e información pública del Ministerio de Vivienda y Urbanismo (MINVU) como el Plan Nacional de Reconstrucción "Chile Unido Reconstruye Mejor"; Análisis de las tendencias de localización Talca 2007; Informe de aplicación de subsidios de densificación media en la Región del Maule, SERVIU Región del Maule; información de subsidios asignados en la ciudad de Talca; información de localización de los conjuntos de construcción de vivienda en terrenos nuevos en la ciudad de 
Talca), información pública del Ministerio del Interior (Diagnóstico del estado de la reconstrucción 2014), datos generados por la Municipalidad de Talca (catastro municipal de daños, Plan de Desarrollo Comunal 2007-2014), datos producidos por los autores en el contexto de su trabajo en investigaciones previas y en curso (catastro de daños en la ciudad de Talca), y datos generados por organizaciones no gubernamentales y comunitarias (mapas de brechas de la Escuela de Líderes de Ciudad ELCI, diagnóstico socio-urbano de villa Las Américas del Programa Territorio y Acción Colectiva TAC, y datos de catastro posterremoto generados por ONG Surmaule y CEUT), e información en prensa de circulación nacional (CIPER) y local (diario El Centro).

Este set de información fue construido en torno a tres objetivos: reconstruir el contexto del caso, describir el caso en sí mismo, e identificar a los actores envueltos en el caso y sus perspectivas e intereses. De este modo, el set de datos fue construido en un proceso circular donde el análisis de la información disponible llevaba a la búsqueda e incorporación de nuevas fuentes, hasta lograr completar el caso.

En primera instancia, se construyó el contexto territorial del caso. Luego, se generó una descripción de la política y el proceso de reconstrucción. Por último, y apoyados en un trabajo de codificación temática, se realizó el análisis de los actores envueltos en el caso y sus perspectivas. Los resultados a continuación siguen este mismo esquema.

\section{Talca y su contexto urbano antes del terremoto}

La expansión urbana de Talca ${ }^{4}$ más allá de su centro histórico se produjo en los últimos 40 años (1970-2010).

Desde los años 80, y en el marco de un modelo neoliberal para comprender y hacer ciudad, se ha impuesto la lógica de utilizar la ciudad como espacio privilegiado para la reinversión de excedentes económicos con altas tasas de rentabilidad (Letelier e Irázabal, 2015). Algunas expresiones de este proceso en Talca son: la llegada masiva de grandes cadenas comerciales; la creciente instalación de nuevas macrotipologías urbanas (centros comerciales, autopistas urbanas, estacionamientos subterráneos) que transforman el paisaje, crean nuevos centros de servicios e impactan diferencialmente el valor de suelo; la proliferación de la edificación en altura con departamentos de más de UF $^{5}$ $2.000^{6}$; el desarrollo de sectores periféricos y periurbanos residenciales y de

\footnotetext{
${ }^{4}$ En el contexto chileno Talca es una ciudad intermedia. Según el Censo de Vivienda y Población de 2012 su población alcanza los 230.000 habitantes (Instituto Nacional de Estadísticas, INE (2012).

${ }^{5}$ La unidad de fomento, UF, es una unidad financiera reajustable de acuerdo con la inflación (medida según el Indice de Precios al Consumidor o IPC). Su valor hoy es cercano a los US\$45.

${ }^{6}$ Estos edificios densifican los perímetros centrales cambiando su carácter morfológico y configuración socioeconómica.
} 
servicios para clases medias altas y altas; y la construcción de grandes sectores de vivienda social en la periferia. En este sentido, la escala de las operaciones inmobiliarias ha crecido, y con esto, también se ha hecho mayor su capacidad de generar transformaciones en la ciudad (Sabatini, Cáceres y Cerda, 2001), y de empujar procesos de diferenciación socioespacial.

El centro histórico de Talca, a diferencia de la periferia, fue construido con lógicas distintas. Procesos de autoconstrucción y desarrollos de vivienda obrera, con décadas de inversión de las familias para mejorar sus viviendas, procesos de movilidad social y de recambio de habitantes, llevaron a que este espacio se constituya hoy día en un lugar en donde conviven calidad urbana y heterogeneidad social. En este sentido, a diferencia de lo que ha ocurrido en la periferia, las zonas centrales mantuvieron su condición de estructura de oportunidades democrática, y su capacidad para generar integración de distintos tipos sociales en el mismo territorio (Rasse y Letelier, 2013).

Así antes del terremoto, junto a la "ciudad neoliberal" de la periferia y los nuevos desarrollos inmobiliarios, coexistía una ciudad anterior, caracterizada por un espacio urbano cuya superficie está contenida en un perímetro que representa aproximadamente el $20 \%$ del total del área urbana, conocida como centro histórico. Esta zona está compuesta por los 15 barrios antiguos de la ciudad, e incluye en el centro del polígono al barrio comercial y de servicios de la ciudad. En el año 2002, vivían 38.078 personas y existían 11.538 viviendas en el centro histórico, de acuerdo con los datos del Censo 2002 (INE, 2002). Según la misma fuente, cerca de 13.000 de sus habitantes eran arrendatarios o allegados.

Dado que hablamos de barrios formados hace ya varias décadas, estos concentran un alto número de habitantes mayores de 60 años, que viven gracias a sus pensiones e ingresos provenientes del subarriendo. Sus bajos niveles de ingreso han llevado al deterioro y pérdida de valor de sus inmuebles. De acuerdo con el CENSO 2002, antes del terremoto, de la totalidad de habitantes, el $56 \%$ eran mujeres, con un $38 \%$ de jefatura de hogar femenina. El $50 \%$ de los habitantes pertenecían a los estratos D y E, siendo el área muy heterogénea socioeconómicamente (INE, 2002).

Por su parte, de 9.454 viviendas, 4.353 (46\%) tenían una materialidad que las hacía muy vulnerables (adobe o tabiquería). El $39 \%$ de las familias, unas 2.500 , eran no propietarias, y el $16 \%$, es decir, aproximadamente 1.000 , eran parte de una sucesión. De acuerdo con los datos del mismo Censo 2002, parte importante de las familias populares del área eran propietarias, y muchos de los arrendatarios subarrendaban en un sitio en que también residía la familia propietaria. Esta estructura de tenencia posibilitaba la retención de la localización por gran parte de las familias populares del área (Rasse y Letelier, 
2013), permitiendo a las familias vivir en un sector con abundancia de oportunidades de diverso tipo, y con espacios públicos de calidad.

De este modo, si bien la ciudad de Talca en las últimas décadas estaba experimentando fuertes transformaciones, su centro histórico mantenía, antes del terremoto, calidad urbana y heterogeneidad y constituía de ese modo una estructura de oportunidades democrática para cerca de 14.000 familias que lo habitaban.

\section{Daños posteriores al terremoto y riesgo de relocalización}

El 27 de febrero de 2010, un terremoto grado 8.8 en la escala de Richter, seguido de un tsunami, afectaron la zona central de Chile, generando graves daños en poblados y ciudades. La región más afectada fue la del Maule.

En Talca, el terremoto del $27 \mathrm{~F}$ afectó principalmente el casco histórico de la ciudad y sus 15 barrios fundacionales, lo que se explica por la concentración de viviendas de adobe en esa zona. Dado que la destrucción se focaliza en el centro de la ciudad, el nivel de daño implica un efecto agregado desde las familias a los barrios y al territorio (Rasse y Letelier, 2013). El registro oficial reconoce solo poco más de 3.500 viviendas dañadas; sin embargo, este dato deja sin catastrar al menos unas 3.000 viviendas de las áreas más afectadas. Mientras el catastro municipal arrojó un porcentaje de daño de $75,1 \%$; aunque estudios posteriores corrigen el dato, arrojando un 80,3\% de daño (Figueroa, 2012).

Tabla 1

Estimación de daños según tipología

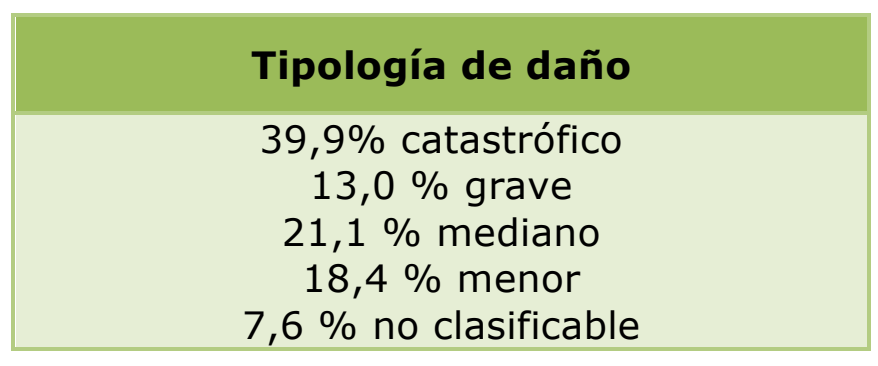

Fuente: CEUT, 2013. Información construida en base a trabajo de campo sobre una muestra representativa de manzanas y lotes de la zona dañada de la ciudad.

La destrucción masiva de viviendas en el casco histórico, producto de una vulnerabilidad física y un riesgo no gestionado, incrementó las vulnerabilidades 
sociales ya presentes en el área. Dadas las políticas de vivienda desarrolladas en los últimos 30 años, donde la localización no es un componente central en la generación de oferta habitacional, el riesgo mayor de expulsión del área lo experimentan las cerca de 3.000 familias que habitaban el centro histórico sin ser propietarias, muchas de ellas con vulnerabilidades agregadas: vejez, jefatura de hogar femenina y pobreza.

A lo anterior se suma que, dados los atributos de la zona central, el precio del suelo había estado incrementándose progresivamente. Antes del terremoto ya era el suelo más caro de la ciudad. Así, el atractivo inmobiliario de algunas zonas del centro se transformó en un nuevo riesgo de pérdida de localización para las familias sin tierra.

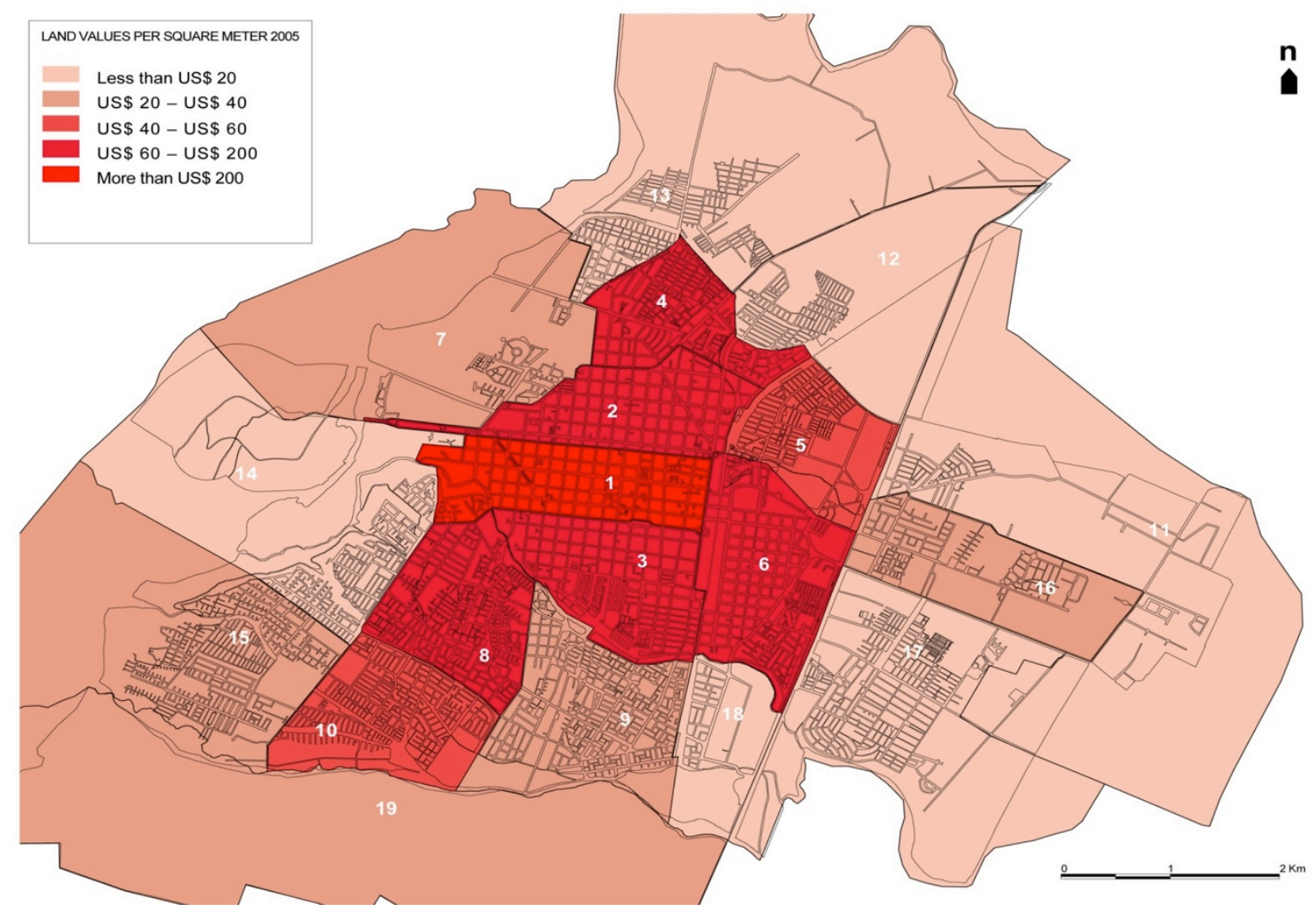

Figura 1. Valores del suelo en Talca.

Fuente: Elaboración propia a partir de Análisis de las tendencias de localización, Talca (Ministerio de Vivienda y Urbanismo, MINVU y Geociudad Consultores, 2007). 


\section{Actores y acciones}

EI modelo de reconstrucción. Para enfrentar la destrucción generada por el terremoto, el Gobierno de Chile decidió enfrentar la situación sin una institucionalidad específica que organizara y dotara a un proceso complejo como este de instrumentos ad hoc. Se optó, en cambio, por utilizar los programas previamente existentes (Rasse y Letelier, 2013), lo que tendría la ventaja de dar velocidad y fluidez al proceso.

Así, si bien existe un documento denominado Plan Nacional de Reconstrucción ${ }^{7}$, se trata de orientaciones de política, sin acompañamiento de instrumental asociado. Algunas de estas orientaciones hacen referencia a aspectos como la identidad, el arraigo, la construcción de comunidad y tejido social, en la práctica los instrumentos estaban enfocados solo a la provisión de viviendas nuevas y a su mejoramiento en el caso de las viviendas antiguas. De este modo, la base de la acción pública en la reconstrucción fueron instrumentos orientados principalmente a financiar la compra de viviendas, proveyendo a las familias de un voucher que les permitiera salir a comprar una vivienda según lo ofrecido en el mercado por ese monto. Específicamente, se podía optar por un subsidio para la compra de una vivienda en un sitio nuevo (orientado a familias sin tierra), por uno para la compra de una vivienda (generalmente prefabricada) en un sitio del que ya se era dueño (para familias propietarias del suelo), o por un subsidio para reparar la vivienda (en casos de viviendas declaradas dañadas, pero habitables). Lo anterior tuvo cinco implicancias:

- Ausencia de visión territorial: el foco principal es proveer de vivienda, sin tomar en cuenta la multidimensionalidad del daño, ni su concentración en ciertas zonas.

- Invisibilización de actores y activos: la respuesta de política pública se produce desde lo que falta (vivienda), pero no desde los activos que las familias tienen.

- Cambio de las formas de habitar: las nuevas viviendas son de tamaños mucho menores a las viviendas previas, lo que impide el subarriendo de habitaciones, o la convivencia con familias o núcleos allegados.

- Expulsión de las familias sin tierra hacia la periferia: la familia extensa, que compartía vivienda, o familias que arrendaban o subarrendaban, se ven forzadas a postular a un subsidio por una vivienda nueva, ya que "no caben" en las soluciones habitacionales. Dado que la política se basa en el subsidio a la demanda, las posibilidades de viviendas nuevas se sitúan en la periferia de la ciudad, donde el suelo es más barato.

\footnotetext{
${ }^{7}$ Elaborado por el Ministerio de Vivienda en 2010 (MINVU, 2010).
} 
- Concentración de la gestión pública en la entrega de subsidios para la construcción o reparación de viviendas y minimización del rol planificador y promotor del sector público. En este sentido, el Estado se entiende a sí mismo como proveedor de vivienda, pero no como responsable de las formas de producción de barrio y ciudad.

Todo esto genera una clara discontinuidad entre el foco socio-territorial y comunitario declarado, y la lógica más bien viviendista que se aplicó en la práctica. Los dos instrumentos diseñados para salvar esta diferencia (el desarrollo de planes de reconstrucción urbana sustentable y los subsidios de patrimonio) tuvieron efectos prácticos bastante acotados en la región.

Los planes de reconstrucción urbana sustentable consistían en planes desarrollados participativamente por actores privados por encargo del sector público. Los privados no solo realizaban el diagnóstico y desarrollaban en plan, sino que también financiaban, a modo de donación, la realización de estos planes. La idea era orientar la reconstrucción de las localidades de acuerdo con estos planes, sin embargo, al no estar vinculados a la institucionalidad ni a partidas presupuestarias, sus propuestas y proyectos no eran vinculantes, y en la práctica la mayor parte de ellos no se ejecutó.

Los subsidios de patrimonio se crearon debido a la dificultad de mantener las características identitarias y patrimoniales de las viviendas con los montos otorgados por los subsidios de reparación. Al comenzar el proceso de reconstrucción de viviendas, se hizo patente que con los instrumentos existentes se generaban viviendas que rompían la continuidad tipológica y las formas de vida de los barrios antiguos o patrimoniales. Para hacer frente a esto, se asignó un monto extra para reconstruir con características patrimoniales (que aunque aumentaba el subsidio, no era suficiente para costear una reconstrucción propiamente patrimonial). En Talca, esto se tradujo en la construcción de viviendas siguiendo la fachada continua que existía antes del terremoto, lo que permitió mantener la imagen exterior de los barrios, pero no la forma de vida: sus características (altura, grosor de los muros, dimensión de las viviendas) eran distintas, y por ende, debían ser habitadas de otra forma. Por esto, su aplicación fue limitada, ya que muchas personas prefirieron intentar rehabilitar sus viviendas anteriores con fondos propios, apoyados en la autoconstrucción.

Análisis de actores. Como queda en evidencia, este modelo implica la relación de actores diversos, con distintos intereses y estrategias, en las acciones de reconstrucción. Entre ellos los siguientes. 
Gobierno central (Ministerio de Vivienda y Urbanismo). El gobierno central se mantuvo en la línea de su modelo que continuaba con la lógica de producción de vivienda centrada en la oferta del sector privado, sin considerar la dimensión territorial (Rasse y Letelier, 2013) ${ }^{8}$. Su interés se centró en agilizar el proceso de entrega de subsidios a los damnificados para que el sector privado pudiera operar rápidamente ejecutándolos, ya que el objetivo era reponer todas las viviendas (independientemente de los procesos de transformación urbana que esto generara) (Rodríguez y Rodríguez, 2013). Así, en la práctica se impulsó un plan que llevó al desplazamiento de las familias allegadas y arrendatarias del centro histórico del Talca, reforzando el patrón de segregación de la ciudad al ofrecerles vivienda en la periferia.

Municipalidad. En un primer momento, cuando el gobierno central aún no hacía públicas sus propuestas de reconstrucción, el alcalde de Talca solicitó al Colegio de Arquitectos un plan de reconstrucción que fue incluso aprobado inicialmente por el Concejo Municipal ${ }^{9}$. Con este plan se anunciaba una alianza virtuosa entre el municipio y la sociedad civil local. Sin embargo, a los pocos días de que el Concejo Municipal aprobara la propuesta, el gobierno central dio a conocer su modelo de reconstrucción, y en conjunto con el propio alcalde decidieron solicitar el plan de reconstrucción a uno de los grandes grupos económicos de Chile, Hurtado Vicuña, específicamente a su Inmobiliaria El Bosque S.A. (Centro de Investigación Periodísticas, CIPER, 2010), siguiendo el proceso que se llevaría en muchas ciudades afectadas, y desconociendo el trabajo ya realizado con la sociedad civil. Al seguir la fórmula propuesta por el gobierno central, el municipio se desligaba de los temas de reconstrucción, desentendiéndose de sus problemáticas y particularmente de la relacionada con los sin tierra. Al mismo tiempo, se quitaba protagonismo a la sociedad civil y a los procesos participativos desde abajo, entregando el liderazgo de los procesos de reconstrucción a los privados, y definiendo que el rol de los habitantes sería el de participar en los espacios definidos, y principalmente, haciéndolo escogiendo solo entre las opciones de vivienda ofertadas.

Inmobiliarios. El modelo de reconstrucción, al mantener los mismos instrumentos utilizados en tiempos normales, presenta a los privados un escenario similar al ordinario, pero con una mayor disponibilidad de subsidios, y por ende, con una demanda por vivienda acrecentada. En este contexto, lo que ocurre es que se aceleran las tendencias que se venían presentando en la ciudad: se desarrolla una fuerte oferta de vivienda económica para familias de bajos recursos en la periferia, y se acrecientan los procesos de gentrificación del centro histórico de Talca, con oferta de vivienda para familias de estratos medios.

\footnotetext{
8 También se evidencia en el Diagnóstico del Estado de la Reconstrucción emanado por el Ministerio del Interior y Seguridad Pública en 2014.

${ }^{9}$ Como consta en el acta de Consejo Municipal del 4 de abril de 2010.
} 
Ambos procesos ya estaban delineados en la ciudad, y lo que hace el terremoto es acelerarlos. Por una parte, la existencia de un gran número de familias damnificadas de bajos recursos y con subsidios de reconstrucción permite a los inmobiliarios aumentar su oferta de vivienda en la periferia ${ }^{10}$. En estos sectores, los inmobiliarios ya tenían suelo comprado con anterioridad en espera de una futura demanda por vivienda, y la contingencia del terremoto les permite apresurar este proceso: el aumento de la demanda por suelo urbano se traduce en un alza del valor de esos terrenos, y en último término, en ganancia.

Por otra parte, algunos barrios del centro histórico, por su localización privilegiada y atributos urbanos, ya comenzaban a mostrar signos de gentrificación. Tras el terremoto, y dada la imposibilidad de los propietarios de reconstruir sus viviendas con las características que tenían, ya a principio de 2013 una importante porción de los propietarios, cerca de un 10\%, había decidido vender sus terrenos, por lo que la oferta de suelo aumentó (CEUT, 2013). Estos fueron comprados por inmobiliarias que, fusionando los predios de dos o tres casas antiguas, generaban un condominio de densidad media o un edificio de departamentos para estratos medios (y así, lograr hacer negocio inmobiliario al aumentar la densidad).

En algunos casos, los nuevos desarrollos en el centro estaban orientados a familias de clase media alta; en otros, a familias de clase media que tenían un subsidio de reconstrucción y que podían solicitar un crédito hipotecario para cubrir la diferencia respecto del valor de la nueva vivienda. Ambos casos, sin embargo, contribuyeron a elevar los precios del suelo en la zona, y cambiar el perfil de los residentes, ampliando la presencia de sectores medios y altos en los barrios centrales (y dificultando el desarrollo de proyectos de vivienda económica en el área, producto del alza en los precios del suelo). Un ejemplo claro de lo anterior es que de los cerca de 1.500 subsidios de densificación orientados inicialmente para damnificados, menos de un $8 \%$ fue utilizado por ellos en el caso de Talca (Servicio de Vivienda y Urbanismo, Serviu, s/f).

Familias damnificadas no propietarias. Las familias damnificadas no propietarias tuvieron un periodo de activación. A mediados de 2010 se conformaron cuatro comités de "Sin Tierra". En conjunto estos comités agrupaba a más de cien familias. Posteriormente estos conformaron una asociación denominada "Agrupación de Comités por una Vivienda en Mi Barrio". Los comités y su agrupación tuvieron una actividad intensa entre el 2010 y el 2011; sin embargo, ya a fines de 2011 la actividad había decrecido notablemente y los comités habían perdido ya la mayor parte de sus

\footnotetext{
${ }^{10}$ Conjuntos de viviendas con Declaración de Impacto Ambiental (DIA), aprobados entre marzo 2010 y julio 2013: http://elci.sitiosur.cl/talca-conjuntos-de-viviendas-con-declaracion-de-impacto-ambiental-diaaprobados-entre-marzo-2010-y-julio-2013
} 
miembros, fundamentalmente porque no existía ninguna evidencia de avance en soluciones habitacionales en los propios barrios y porque algunos de sus integrantes habían ido adquiriendo soluciones habitacionales de manera individual. En este sentido, la urgencia de contar con una vivienda, junto con la amplia disponibilidad de oferta de vivienda nueva en sitios periféricos (y por el contrario, la enorme dificultad de conseguir vivienda en el lugar en que se habitaba) minó la fuerza de las organizaciones que buscaban una solución en el propio barrio. Solo uno de los comités logró una solución relativamente adecuada: el comité San Pelayo del Barrio Seminario con el proyecto "Los Maitenes".

Familias damnificadas propietarias. Las familias damnificadas que sí eran dueñas de la propiedad que habitaban tampoco enfrentaron una oferta de política pública adecuada a sus necesidades. En primer lugar, muchas de las familias ocupaban sus viviendas de forma irregular, sin haber hecho los traspasos, posesiones efectivas, etc. que los acreditaban como propietarios. Esto era requisito para la obtención de cualquier beneficio. Adicionalmente, se trataba de familias que no eran usuarios habituales de los programas sociales, ya que muchos eran personas de tercera edad, de clase media empobrecida, que no tenían costumbre ni conocimientos para relacionarse de manera eficaz con el aparato estatal. En este sentido, el proceso se les representaba como engorroso o incluso incomprensible, y requerían de ayuda para poder gestionar sus beneficios. No todos los damnificados lograron postular con éxito a los beneficios en los plazos estipulados (Valdivieso, 2013).

El interés principal de este grupo de familias era recuperar su vivienda lo más parecida posible a como era antes del terremoto. Este proyecto se vio truncado, ya que las viviendas originales eran muy grandes, y el dinero del subsidio era bastante menor a lo requerido para reconstruir manteniendo esta característica. En el caso de las familias con viviendas irreparables, las viviendas nuevas a las que podían optar eran totalmente diferentes a las que tenían antes: se trataba de viviendas de la mitad de metraje o menos; en una materialidad distinta (sin la aislación que les proveía el adobe), y que apenas cubrían un trozo del sitio (a diferencia de la vivienda original, que cubría todo el frontis). Por este motivo, muchos prefirieron sustraerse del proceso.

En el caso de las familias con viviendas que requerían reparaciones, estas eran mucho más costosas que los montos ofrecidos por el subsidio. Esto, sumado a las dificultades para acceder a los subsidios, llevó a que la mayoría optara por reconstruir con fondos propios, en plazos más largos (Rasse y Letelier, 2013). En este sentido, los intereses y racionalidad de los damnificados propietarios eran divergentes respecto de la propuesta de política pública que buscaba abordarlos (Valdivieso, 2013), lo que llevó en la práctica a que el proceso de reconstrucción de los barrios centrales se desplegara de forma lenta, y que incluso hoy sus logros sean bastante modestos, ya que el motor de la reconstrucción en estos barrios fue más bien la recuperación de las viviendas 
con fondos de los propios habitantes. A dos años del terremoto un $40 \%$ de los sitios con daño no habían sido intervenidos; un año después, a principios de 2013, el porcentaje era de 38\% (CEUT, 2013).

Organizaciones de la sociedad civil. Las organizaciones de la sociedad civil (OSC), ONGs, Colegio de Arquitectos y el Movimiento Nacional por una Reconstrucción Justa, entre otras, desarrollaron un fuerte trabajo de generación de análisis y propuestas del proceso de reconstrucción y difusión pública de sus alcances y deficiencias. Durante el 2010 y hasta mediados de 2011 se produjeron las articulaciones más fuertes entre los damnificados sin tierra, sus organizaciones y esta red de OSC. Pese a los esfuerzos de la sociedad civil, los espacios de diálogo con la autoridad fueron sumamente acotados y poco fructíferos y no se logró abrir alternativas para el impulso de proyectos de integración social. Las OSC siguieron desarrollando una labor en el ámbito de la problematización de la reconstrucción, pero como se ha señalado, las familias damnificadas, al constatar la ausencia de voluntad de parte de las autoridades para avanzar en su agenda, comenzaron a buscar soluciones que el modelo les ofreció.

Tabla 2

Caracterización de entes involucrados

\begin{tabular}{|c|c|c|}
\hline $\begin{array}{c}\text { Entes } \\
\text { involucrados }\end{array}$ & Intereses & Estrategias/Acciones \\
\hline $\begin{array}{c}\text { Gobierno/Minvu/ } \\
\text { Serviu }\end{array}$ & $\begin{array}{l}\text { - Agilizar la entrega de } \\
\text { subsidios y de viviendas. } \\
\text { - Relevar el rol del sector } \\
\text { privado en la } \\
\text { reconstrucción. }\end{array}$ & 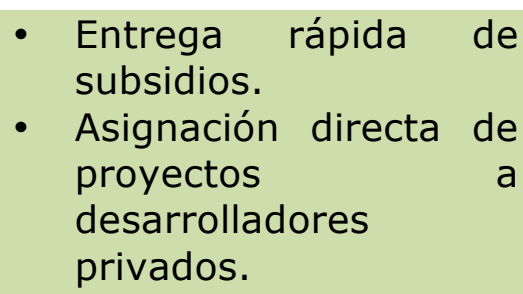 \\
\hline Municipalidad & $\begin{array}{l}\text { - Dejar atrás el terremoto } \\
\text { y retomar su agenda } \\
\text { municipal. } \\
\text { Colaborar con el } \\
\text { gobierno central. }\end{array}$ & $\begin{array}{l}\text { - Evitar responsabilidad } \\
\text { directa en obras de } \\
\text { reconstrucción. } \\
\text { - Reforzar agenda extra } \\
\text { reconstrucción. }\end{array}$ \\
\hline $\begin{array}{c}\text { Sector } \\
\text { inmobiliario } \\
\text { dominante } \\
\text { (Empresas } \\
\text { constructoras/in } \\
\text { mobiliarias/EGIS) }\end{array}$ & $\begin{array}{l}\text { - Potenciar } \\
\text { inmobiliario en la zona } \\
\text { central con oferta de } \\
\text { mayor valor al } \\
\text { preexistente. } \\
\text { - Desarrollar proyectos } \\
\text { urbanos en la periferia }\end{array}$ & $\begin{array}{l}\text { - Desarrollo rápido de } \\
\text { proyectos de vivienda } \\
\text { económica en la } \\
\text { periferia. } \\
\text { Desarrollo } \\
\text { condominios de } \\
\text { estratos medios y }\end{array}$ \\
\hline
\end{tabular}




\begin{tabular}{|c|c|c|}
\hline & $\begin{array}{l}\text { de la ciudad (urbanizar y } \\
\text { vender). }\end{array}$ & $\begin{array}{l}\text { medio altos en el } \\
\text { centro histórico. } \\
\text { - } \begin{array}{l}\text { Presión para la } \\
\text { generación }\end{array} \\
\begin{array}{l}\text { subsidios de } \\
\text { aplicarse en los } \\
\text { proyectos de la zona } \\
\text { centro. }\end{array}\end{array}$ \\
\hline $\begin{array}{c}\text { Familias } \\
\text { damnificados }\end{array}$ & $\begin{array}{l}\text { - Obtener una solución } \\
\text { habitacional, } \\
\text { preferencia en el propio } \\
\text { barrio. }\end{array}$ & $\begin{array}{l}\text { - Formación de comités, } \\
\text { búsqueda de apoyo } \\
\text { político, autogestión. } \\
\text { - Autofinanciamiento de } \\
\text { las obras de } \\
\text { reconstrucción y } \\
\text { reparación. }\end{array}$ \\
\hline $\begin{array}{l}\text { Organizaciones de } \\
\text { la Sociedad Civil } \\
\text { (ONGs, } \\
\text { Movimientos, etc.) }\end{array}$ & $\begin{array}{l}\text { - Defender el derecho a la } \\
\text { localización de las } \\
\text { familias sin tierra. }\end{array}$ & 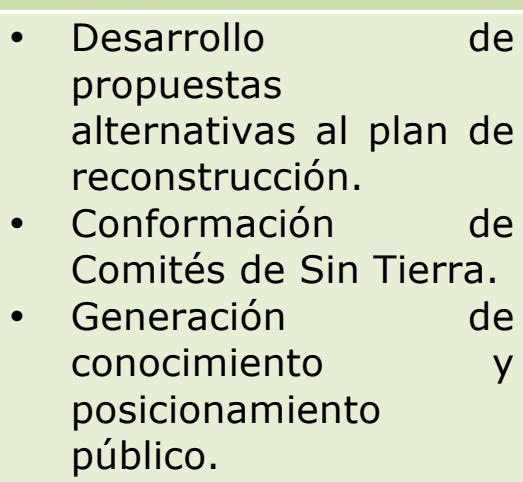 \\
\hline
\end{tabular}

Como puede observarse, el único interés transversal a los actores es la provisión rápida de vivienda. De hecho, en este punto, la política pública se alinea por completo con los intereses de los inmobiliarios. Así, la provisión de soluciones habitacionales era empujada desde los actores con mayor poder, por lo que pronto hubo una oferta importante de vivienda disponible, pero en la periferia: la búsqueda de mantención de localización fue relevada por actores sin acceso al poder (las propias familias y las organizaciones de la sociedad civil), pero sin lograr concretarse. El gobierno local, que podría haber tomado las demandas de la sociedad civil, decidió restarse del proceso. Como consecuencia, los sin tierra fueron tratados como damnificados desterritorializados, es decir, se invisibilizó toda relación de las familias con el territorio, su arraigo y sus redes.

Desenlace. En Talca se asignaron cerca de 4.500 subsidios que permitían a una familia damnificada obtener una nueva vivienda. De ellos, 1.000 fueron destinados para propietarios, mientras otros 3.500 subsidios 
fueron ofertados a familias no propietarias. Estos últimos subsidios eran potencialmente utilizables para la radicación de damnificados sin tierra en sus propios barrios, combinándolos con el subsidio de integración social ${ }^{11}$, y con el apoyo de las organizaciones sociales. Desarrollar conjuntos de vivienda que combinaran vivienda para familias damnificadas de bajos recursos con vivienda orientada al mercado hubiera permitido pagar el alto valor del suelo (gracias a las unidades de mercado), y al mismo tiempo, lograr la permanencia de los damnificados sin tierra en el centro histórico.

Esto fue, de hecho, lo que ocurrió en el caso del conjunto "Los Maitenes", correspondiente al comité San Pelayo del barrio Seminario. Fue clave en este caso la presencia en el barrio de La Provincia, una empresa constructora local de propiedad de una de las familias más antiguas del sector. La vinculación del comité con esta empresa, sumado al trabajo de promoción de la ONG Surmaule y el aporte en el diseño del proyecto arquitectónico de la ONG Reconstruye, permitió el desarrollo del primer proyecto posterremoto en todo Chile con viviendas en densidad media en zonas céntricas y con integración social.

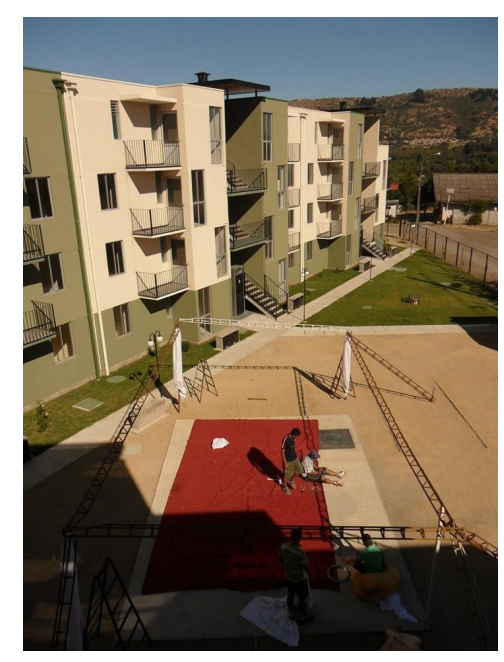

Foto 1. Conjunto habitacional "Los Maitenes".

Fuente: Comité de vivienda San Pelayo.

\footnotetext{
${ }^{11}$ Subsidio que se asigna a aquellos compradores de estratos medios dispuestos a comprar una vivienda en un conjunto que incluye vivienda social.
} 
En este caso, se combinaron los subsidios disponibles (de reconstrucción; de integración social y para familias de clase media) para generar un conjunto que permitiera, por una parte, dar solución habitacional a los damnificados sin tierra, y por otra, atraer a familias de clase media, manteniendo la diversidad social preexistente en el barrio. El conjunto quedó constituido por dos edificios de 4 pisos con 36 viviendas de $55 \mathrm{~m}^{2}$, de las cuales 20 han sido destinadas a damnificados por el terremoto ( $y$ por lo tanto, sujetas a subsidio), siendo comercializadas las restantes utilizando subsidios para familias de clase media ${ }^{12}$, convirtiéndolo en un proyecto de integración social (al incorporar familias de distintos estratos). Es evidente, en este caso, el valor de este tipo de solución para la ciudad en términos en la reducción de las tendencias de segregación social previas al terremoto, y para las familias, al permitirles conservar el la localización en el centro como un activo de integración social a la ciudad.

El proceso para lograr este proyecto no fue sencillo. Micaela Torres, presidenta del comité San Pelayo, relata:

... nadie creía mucho en esto porque empezamos a contar la plata y no alcanzaba para nada... Las constructoras generalmente marginan el $70 \%$ de las utilidades, pero en estos proyectos no puedes marginar tanto, entonces nadie nos pescaba. Pero en Reconstruye hicieron este proyecto de integración especialmente para nosotros: nuestro edificio va a tener 36 departamentos, 20 van a ser para los del comité y los otros se van a vender a cualquier persona y de ahí se van a generar las utilidades para interesar a la constructora y para poder pagarle al dueño del terreno (Levantemos ciudadanía, 2012).

La generalidad de los casos fue otra muy distinta. Un ejemplo de ello son los conjuntos Villa El Parque, donde se creó un nuevo conjunto habitacional de 600 familias sin historia común y con viviendas de mínimo estándar.

\footnotetext{
${ }^{12}$ En particular, se refiere a los Susidios Habitacionales del tipo D.S. N40 y DS1, los cuales son entregados por el Ministerio de Vivienda y Urbanismo.
} 


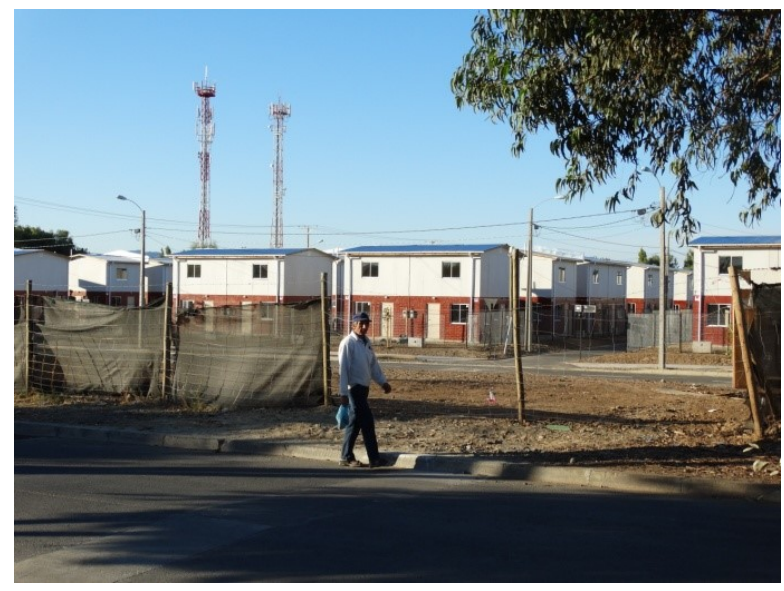

Foto 2. Conjunto Villa el Parque.

Fuente: Programa Territorio y Acción Colectiva.

Cuando se observa el panorama general, se concluye que la totalidad de los proyectos con subsidio de construcción en nuevos terrenos fueron localizados fuera del casco histórico, lo que implicó el traslado de las familias a la periferia. A esto se suma que solo el $7 \%$ de los subsidios de densificación urbana (aplicados en el centro) fueron usados por familias damnificadas. Es decir, los subsidios que hubieran permitido retener a las familias en el centro, en la práctica no fueron utilizados para ello (ya que las unidades construidas eran de mayor valor, y los damnificados de menores ingresos no podían optar a crédito hipotecario para cubrir la diferencia). Esto evidencia tanto la pérdida de localización de las familias sin tierra del centro, como el cambio de la composición social del mismo, que aumenta su nivel socioeconómico promedio. 


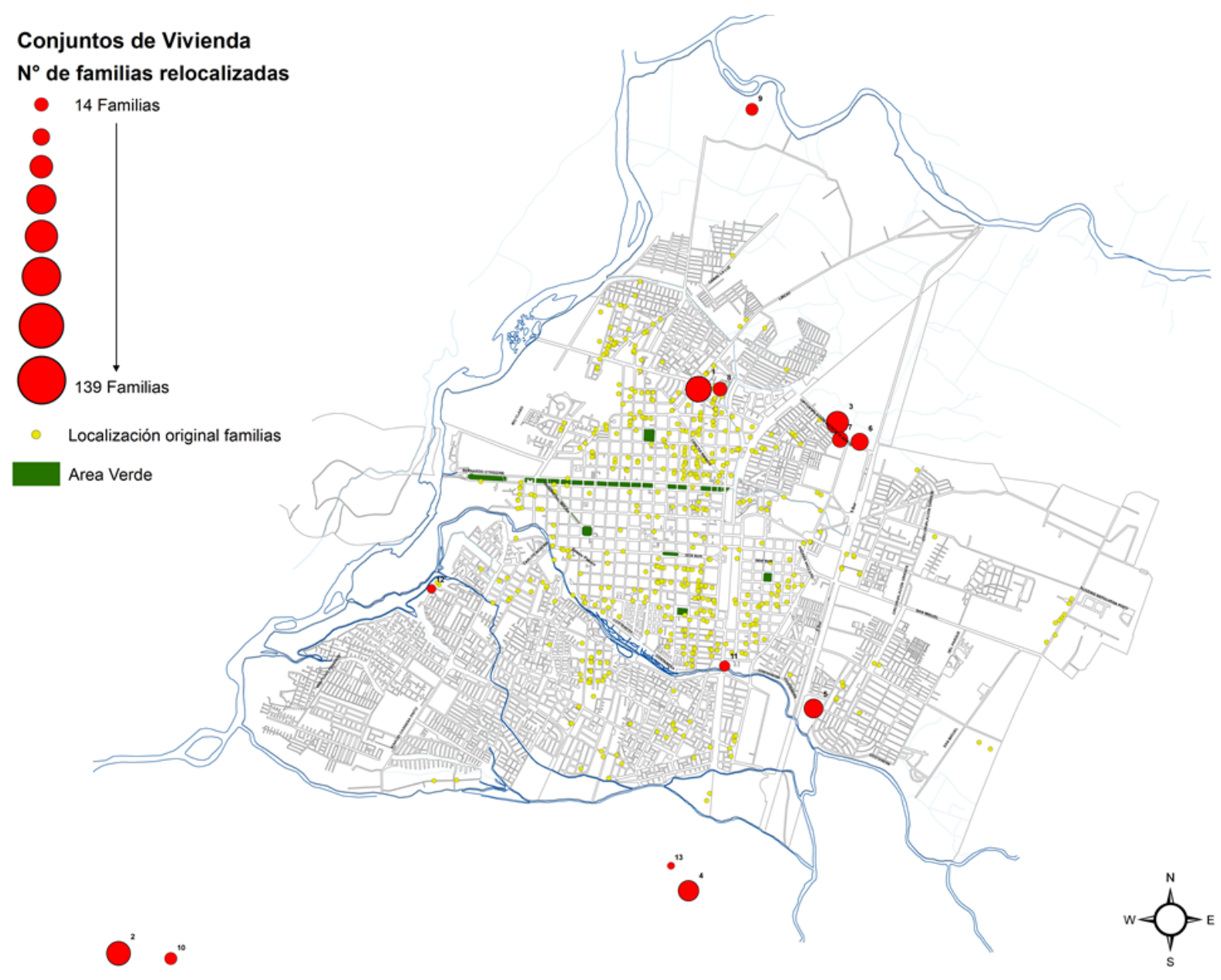

Figura 2. Localización original de familias relocalizadas posterremoto. Fuente: Elaboración propia sobre la base de datos Serviu Región del Maule de proyectos CNT de reconstrucción en la comuna de Talca.

En el intertanto, el $38 \%$ de los lotes correspondientes a vivienda con daño grave en el centro de la ciudad seguía vacío al 2013 y continúa en una situación muy similar a cuatro años del terremoto.

El factor clave en este resultado no fue el tipo instrumentos disponibles, ya que como se ha evidenciado, lo disponible podía ser conjugado para lograr la retención de los damnificados sin tierra en el centro. Lo fundamental fueron los supuestos sobre el rol del Estado y los privados en el desarrollo urbano subyacentes al modelo de reconstrucción: predominó la idea de un Estado que debía facilitar la participación de los privados en las iniciativas de reconstrucción, y sobre todo, permitir el acceso al mercado de vivienda a quienes no podían hacerlo por sí solos. Esto deja en manos de los privados el 
proceso de reconstrucción, tomando el Estado un rol más bien financista, y dando centralidad en el proceso al mercado de vivienda.

Como consecuencia, todo lo extra a la provisión de vivienda (la recuperación de los barrios o el derecho a la localización) no fue considerado. Asimismo, los programas que apelaban a otros elementos, como el subsidio patrimonial (para la reconstrucción con fachada continua) o el de densificación urbana, tuvieron una muy acotada aplicación, o bien, fueron utilizados con otro sentido.

A lo anterior se sumó la omisión activa de los actores políticos locales, quienes podrían haber sido gravitantes para cambiar la posición del Gobierno. Actores locales con una posición de apoyo activo hubieran logrado movilizar al Minvu y Serviu a desarrollar estrategias vinculadas a la radicación de las familias, por ejemplo, para impulsar más Proyectos de Integración Social (PIS), en conjunto con constructoras de pequeño y mediano alcance interesadas. Esta era la posición de la agrupación de Comités de Sin Tierra, evidenciada en un fragmento de la carta que el 16 de noviembre de 2010 la agrupación le envió a la Ministra de Vivienda de ese entonces:

Para el logro de nuestro objetivo, que es quedarnos en nuestros barrios, requerimos voluntad y creatividad de todos los actores involucrados, autoridades y vecinos. Proponemos que los instrumentos hoy existentes puedan ser flexibilizados. Por ejemplo, que el subsidio de habilitación de hasta UF 200, destinado a compra y habilitación de terreno, sea entregado en su totalidad y que su uso esté destinado a compra de terreno. Esto permitiría la compra de terrenos en nuestros barrios.

Sin embargo, para tener actores políticos locales empoderados se requería una ciudadanía convencida de la localización como derecho. Si bien diversas organizaciones desarrollaron una tarea fuerte en esta línea, la presión ciudadana no llegó a niveles que pudieran mover a los actores políticos. Los damnificados sin tierra tomaron una actitud más pasiva, sin generar acciones masivas de presión hacia la autoridad. Lo contrario sucedió con otros casos emblemáticos en Talca, donde la ciudadanía hizo una defensa más activa. En el caso de los sin tierra, la demanda fue siempre difusa y no se logró construir una masa crítica capaz de mover a la clase política de su posición de neutralidad, omisión activa, u oposición pasiva. Así, la perseverancia del Gobierno en las soluciones definidas desde un principio, y el desinterés en promover otras alternativas, debilitó fatalmente al incipiente movimiento social que se había originado. 


\section{Conclusiones}

Como queda en evidencia, contar con una política de reconstrucción que (a) se centró en la provisión de vivienda; (b) dejó el liderazgo a los privados; y (c) se basó en subsidio a la demanda, tuvo un importante efecto de desplazamiento de las familias damnificadas sin tierra del centro a la periferia, llevando a la intensificación de las tendencias de segregación de la vivienda social y gentrificación del centro. En este sentido, más allá del éxito o fracaso de la política en términos de reconstrucción, es importante tener en cuenta sus consecuencias más profundas en la configuración de la ciudad de Talca.

Por otra parte, el caso revisado permite relevar algunas claves para las políticas públicas vinculadas con los procesos de recuperación posdesastre y en general a la gestión urbana y del territorio.

En primer lugar, se evidencia la importancia que tiene la existencia de marcos de política que orienten la acción pública en procesos de recuperación posdesastre. En el caso analizado, el marco de política dejó el liderazgo de los proceso a los privados, lo que minimizó el rol de Estado, incluso a pesar de las recomendaciones internacionales como las que ha hecho el Banco Mundial. Esto tiene como consecuencia no solo la incapacidad para observar y problematizar los fenómenos urbanos emergentes, sino que también implica la desaparición del debate acerca del bien común y de lo que se desea para la ciudad.

Una segunda cuestión tiene relación con rol de las municipalidades. Las experiencias de reconstrucción posterior al 27 de febrero muestran que distintos municipios asumieron posiciones diferentes respecto del proceso. Algunas se involucraron con intensidad, otras fueron simples espectadores, como la municipalidad de Talca. Se entiende que la mayoría de las tareas vinculadas con la reconstrucción de viviendas no le compete a los gobiernos locales, lo cual es necesario revisar; pero por otro lado, la municipalidad, que tiene como rol constitucional el asegurar la participación de los habitantes en el desarrollo de la comuna, debe ser un actor clave en los procesos de recuperación posdesastre. Dejar a discreción del alcalde involucrarse o no, significa dejar a las comunidades impedidas de incorporar sus intereses en el proceso de solución.

En tercer lugar se destaca la cuestión de la localización. En Chile existe un subsidio que complementa el de acceso a la vivienda y que en teoría ayuda a mejorar la localización de los nuevos conjuntos de vivienda pública. Sin embargo, su implementación: (a) exige condiciones muy básicas para su aplicación, es posible aplicarlo prácticamente en cualquier parte de la ciudad, por lo que no discrimina entre buena y mala localización y (b) al añadirse al 
subsidio base, como puede ser utilizado en cualquier parte de la ciudad, en la práctica significa un aumento del subsidio, el que rápidamente es capturado en su totalidad por el mercado de suelo (sin necesariamente posibilitar mejores localizaciones). Este problema debe enfrentarse de dos maneras simultáneas: una forma es modificando la política pública de suelo, asegurando la disposición de un stock para vivienda pública en sectores no periféricos; la segunda, menos estructural, pero igualmente necesaria, es fortalecer el rol de las organizaciones que demandan vivienda, incrementar su capacidad de control sobre el proceso de producción de soluciones y su conciencia acerca de la localización como un derecho.

En cuarto lugar está el fortalecimiento de la sociedad civil y las comunidades. En el caso analizado se observa que las organizaciones civiles que se comprometieron con el control social del proceso de reconstrucción no lograron permear la política pública. Esto implica la necesidad de fortalecer las competencias de las organizaciones para actuar en el espacio público, apoyar el trabajo a través de nuevas vías de financiamiento y definir marcos de participación más efectivos.

Finalmente, se concluye que producto de la invisibilización del problema de los sin tierra se genera un nuevo problema: los nuevos conjuntos de vivienda pública localizados en general en la periferia de las ciudades más dañadas. La experiencia indica que cuando se crean conjuntos de vivienda en la periferia, de composición social y económica homogénea, sin los adecuados servicios y sin conocimiento previo entre los habitantes, existen posibilidades muy altas de deterioro urbano. De hecho, los nuevos conjuntos están en zonas que ya empezaban a mostrar consecuencias negativas de segregación residencial, y ante los cuales la política pública aún no logra generar una respuesta eficaz.

Las políticas de vivienda han puesto el acento en producir soluciones masivas. En el caso "Los Maitenes", el incentivo inicial no lo puso el Estado, sino que fue la motivación intrínseca de actores sociales comprometidos con el desarrollo de una ciudad más inclusiva la que generó el empuje necesario; sin embargo, la respuesta habilitadora del Gobierno en última instancia facilitando la construcción del PIS permite ejemplificar la sinergia que se puede crear cuando todos los actores suman esfuerzos para posibilitar la radicación de la población en su territorio. Lo relevante parece ser que existan espacios para acoger la diversidad de intereses y opciones que vienen desde la ciudadanía, y fortalecer las organizaciones sociales que hacen posible este tipo de proyectos.

En este marco, es necesario reflexionar cómo las políticas posdesastre, y en general las políticas de vivienda, generan estímulos para que los prestadores privados desarrollen propuestas que no solo provean un techo, sino que 
también mejoren la calidad de vida de las familias y hagan eco de la idea de que el derecho a la vivienda adecuada es crítico para abrir la puerta a un conjunto de otros derechos, entre ellos, el derecho a la localización y en último término, a la ciudad.

En el caso de procesos de recuperación posdesastre, el resguardo de este derecho pasa necesariamente por construir una institucionalidad y un marco de política que esté por encima de las motivaciones de corto plazo de los gobiernos de turno. 


\section{Referencias bibliográficas}

Centro Estudios Urbano Territoriales, CEUT. (2013). Catastro avance de la reconstrucción. Recuperado de http://www.ceut.cl/

Centro de Investigación Periodística, CIPER. (2010). Nuevo terremoto en Talca: el desembarco del grupo Hurtado Vicuña y la presión inmobiliaria que amenaza a los damnificados. Recuperado de http://ciperchile.cl/2010/04/27/nuevo-terremoto-en-talca-eldesembarco-del-grupo-hurtado-vicuna-y-la-presion-inmobiliaria-queamenaza-a-los-damnificados

Concha, C. y Rasse, A. (2014). La ruta de las caletas del Maule. Sobre la articulación entre sector público, privado y sociedad civil en los procesos de reconstrucción posterremoto. Revista de Geografía Norte Grande, 59, 165-184. http://dx.doi.org/10.4067/S0718-34022014000300010

Davidson, M. \& Lees, L. (2010). New build gentrification: Its histories, trajectories, and critical geographies. Population, Space and Place, 16, 395-411. http://dx.doi.org/10.1002/psp.584

Davoudi, S. (2012). Resilience: A bridging concept or a dead end? Planning theory and practice, 13(2), 299-308. http://dx.doi.org/10.1080/14649357.2012.677124

Ducci, M. (1986). El terremoto de México y las tareas de reconstrucción. ¿Una lección para América Latina? Eure, 13(38), 35-45. Recuperado de: http://www.eure.cl/index.php/eure/article/view/969

Figueroa, K. (2012). Propuesta de ordenanza local. Memoria explicativa, seccional Barrio Santa Ana (Memoria de título). Escuela de Arquitectura, Universidad de Talca.

Instituto Nacional de Estadísticas, INE. (2002). Censo 2002. Recuperado de http://www.ine.cl/cd2002/sintesiscensal.pdf

Instituto Nacional de Estadísticas, INE. (2012). Censo de vivienda y población de $2012.2 \quad$ Recuperado de http://www.ine.cl/canales/menu/publicaciones/compendio_estadistico/c ompendio_estadistico2012.php

Inzulza, J. (2014). La recuperación del diseño cívico como reconstrucción de lo local en la ciudad intermedia: el caso de Talca, Chile. Revista AUS, 15, 4-8. http://dx.doi.org/10.4206/aus.2014.n15-02 
Inzulza, J. y Díaz, I. (2016). Desastres naturales, destrucción creativa y gentrificación: estudios de casos comparados en Sevilla (España), Ciudad de México (México) y Talca (Chile). Revista de Geografía Norte Grande, 64, 109-128. http://dx.doi.org/10.4067/S071834022016000200008

Lechner, S., Jacometti, J., McBean, G., \& Mitchison, N. (2016). Resilience in a complex world - Avoiding cross-sector collapse. International Journal of Disaster Risk Reduction, 19, 84-91. http://dx.doi.org/10.1016/j.ijdrr.2016.08.006

Letelier, F. \& Irázabal, C. (2015). Earthquake reconstruction and disaster fix in Talca, Chile. Journal of Planning Education and Research (JPER). Manuscript ID: JPER-2013-118.R1.

Letelier, F. y Boyco, P. (2013). Talca a tres años del terremoto: aprendizajes colectivos para la acción en la ciudad. Santiago: Ediciones Sur. Recuperado de: http://www.sitiosur.cl/r.php?id=933

Levantemos ciudadanía. (2012). Entrevista a Micaela Torres, 26 de febrero de $2012 . \quad$ Recuperado http://www.levantemosciudadania.cl/archives/2785

Ministerio de Vivienda y Urbanismo, Minvu y Geociudad Consultores. (2007). Análisis de las tendencias de localización, Talca. https://is.gd/LtwKvE

Ministerio de Vivienda y Urbanismo, Minvu. (2010). Plan Nacional de Reconstrucción. Recuperado de http://www.minvu.cl/opensite_20100827194336.aspx

Rasse, A. y Letelier, F. (2013). El proceso de reconstrucción de viviendas en el centro de Talca: fotografía a dos años de la catástrofe. Revista INVI, 28(77), 139-164. Recuperado de: http://revistainvi.uchile.cl/index.php/INVI/article/view/728

Rodríguez, A. y Rodríguez, P. (2013). Misión cumplida señor presidente. Informe de ciudad y reconstrucción. Revista Barómetro de Política y Equidad, 6(9), 176-197. Recuperado de http://fes.cl/media/pdf/barometro06.pdf

Sabatini, F., Cáceres, G., y Cerda, J. (2001). Segregación residencial en las principales ciudades chilenas: tendencias de las tres últimas décadas y posibles cursos de acción. Eure, 27(82), 21-42. http://dx.doi.org/10.4067/S0250-71612001008200002 
Servicio de Vivienda y Urbanismo, Serviu. (s/f). Informe de aplicación de subsidios de densificación media en la Región del Maule. Recuperado de http://www.minvu.cl/opensite_20111122094957.aspx

Shaw, K. (2012). "Reframing" resilience: Challenges for planning theory and practice. Planning Theory and Practice, 13(2), 308-312. http://dx.doi.org/10.1080/14649357.2012.677124

Valdivieso, M. (2013). Modelo de reconstrucción, representaciones y estrategias vecinales pos terremoto en barrio Santa Ana de Talca (Tesis de grado en Antropología). Universidad Academia de Humanismo Cristiano, Santiago de Chile.

Wamsler, C. (2007). Integrando la gestión del riesgo, planificación urbana y vivienda social: lecciones de El Salvador. Revista INVI, 22(59), 93-114. Recuperado de: http://revistainvi.uchile.cl/index.php/INVI/rt/printerFriendly/290/918 